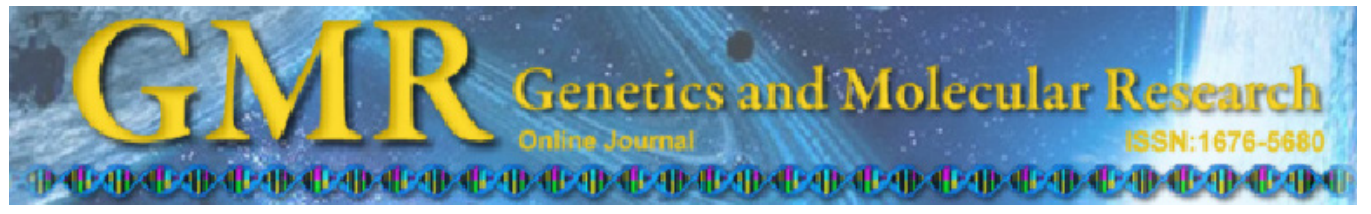

\title{
Novel splice variants of the bovine PCK1 gene
}

\author{
Z.B. Zhangi, ${ }^{1 *}$, W. Zhang ${ }^{2 *}$, R.L. Li $^{2}$, J.B. Li ${ }^{2}$, J.F. Zhong ${ }^{2}$, Z.S. Zhao ${ }^{1}$ and \\ J.M. Huang ${ }^{1}$ \\ ${ }^{1}$ College of Animal Science and Technology, Shihezi University, \\ Shihezi, China \\ ${ }^{2}$ Laboratory of Molecular Genetics and Breeding, Dairy Cattle Research Center, \\ Shandong Academy of Agricultural Sciences, Jinan, Shandong, China \\ *These authors contributed equally to this study. \\ Corresponding authors: Z. Zhao / J. Huang \\ E-mail: zhaozongsh@shzu.edu.cn / huangjinm@sina.com
}

Genet. Mol. Res. 12 (3): 4028-4035 (2013)

Received March 2, 2013

Accepted July 29, 2013

Published September 27, 2013

DOI http://dx.doi.org/10.4238/2013.September.27.4

ABSTRACT. Phosphoenolpyruvate carboxykinase 1 (PCK1), also
named PEPCK-C, is a multiple-function gene that is involved in
gluconeogenesis, glyceroneogenesis, reproduction, female fertility,
and development of obesity and diabetes. How its many functions are
regulated was largely unknown. Therefore, we investigated mRNA
expression and possible splice variants of $P C K 1$ by screening cDNA
in nine tissues from Holstein bulls and cows. PCK1 mRNA was highly
expressed in the liver, kidney, ovary and testis; expression levels were
low in the heart, spleen, and lung tissues. Expression of this gene was
not detected in skeletal muscle. This led to the discovery of five novel
bovine splice variants, named $P C K 1-A S 1-P C K 1-A S 5$. In $P C K 1-A S 1$,
51 nucleotides in the interior of exon 2 were spliced out. In $P C K 1-$
$A S 2$, exons 2 and 3 were altered by the alternative 3 ' and 5 ' splice sites,
respectively. $P C K 1-A S 3$ was truncated from the $3^{\prime}$ end of exon 2 to the
$5^{\prime}$ end of exon 4 . In $P C K 1-A S 4$, exon 5 was completely spliced out.
In $P C K 1-A S 5$, exons 5 and 6 and the 5 ' end of exon 7 were spliced
out. These splice variants $(P C K 1-A S 1-P C K 1-A S 5)$ potentially encoded 
shorter proteins $(605,546,373,246$ and 274 amino acids, respectively), when compared to the complete protein (622 amino acids). Considering the functional domains of the PCK1 protein, it is likely that these splice variants considerably affect the function of this protein; alternative splicing could be one of the mechanisms by which the diverse functions of PCK1 are regulated.

Key words: Alternative splicing; PEPCK gene; Liver; Testis; Bull; Cow

\section{INTRODUCTION}

Phosphoenolpyruvate carboxykinase (PEPCK) is a rate-limiting enzyme of gluconeogenesis that catalyzes the formation of phosphoenolpyruvate from oxaloacetate in the liver and renal cortex. Therefore, the enzyme has been thought to be essential in glucose homeostasis. PEPCK is involved in glyceroneogenesis in liver and white and brown adipose tissue (Hanson and Patel, 1994). PEPCK is synthesized as two isoforms, cytosolic (PEPCK-C, also called PCK1) and mitochondrial (PEPCK-M, also called PCK2). The two isozymes are encoded by separate nuclear genes. All vertebrate species have both PEPCK isoforms but express them at different levels. For example, most mammals have similar activities of both PCK1 and PCK2 in their liver, while the rat and mouse have $90-95 \%$ PCK1 and birds have $100 \%$ PCK2 (Chakravarty and Hanson, 2007). There is accumulating evidence that $P C K 1$ is a multi-function gene that can play important roles in physiological processes and diseases, such as, glucose and triglyceride/fatty acid metabolism in the liver, kidney and adipose tissues, and obesity and diabetes (Chakravarty et al., 2005). Moreover, the PEPCK gene is also involved in aggressive behavior, feeding, extended longevity and reproductive capacity, which was evidenced by overexpressing PCK1 in skeletal muscle in mice (Hakimi et al., 2007; Hanson and Hakimi, 2008). $P E P C K$ of mycobacterium tuberculosis has been shown to trigger cell-mediated immune response by increasing the activity of cytokines, and PEPCK may be a promising new subunit vaccine candidate for tuberculosis (Liu et al., 2006). Interestingly, another study suggests that the role that $P E P C K$ plays in gluconeogenesis may be mediated by the citric acid cycle, the activity of which was found to be directly related to PEPCK abundance. Mouse PEPCK levels alone were not found to be highly correlated with gluconeogenesis in the liver (Burgess et al., 2007). However, PEPCK is expressed not only in the liver, kidney and adipose tissues but also in other mammalian tissues, including the small intestine, colon, mammary gland, adrenal gland, lung and muscle, which is not associated with gluconeogenesis or glyceroneogenesis, a finding that is not explained by this understanding (Croniger et al., 2002). Therefore, the role of PEPCK may be more complex and may involve more factors than previously reported.

Alternative splicing (AS) is an important mechanism for regulating gene expression in cells (Maniatis and Tasic, 2002). The protein and mRNA isoforms produced by alternative processing of primary RNA transcripts may differ in function, structure, localization or other properties (Matlin et al., 2005). Alternative splicing has been proposed as a primary driver of the evolution of phenotypic complexity in mammals for it is known to affect more than half of all human genes (Johnson et al., 2003). About $21 \%$ of all bovine genes $(21,755)$ are alternatively spliced (Chacko and Ranganathan, 2009). 
Bovine $P C K 1$ gene is located on chromosome 13, contains 10 exons and 9 introns and encodes 622 amino acids (Agca et al., 2002). We predicted that the PCK1 gene may exist some variants because multiexon genes have high probability of expression via alternative splicing. Although the mechanisms responsible for the tissue-specific and hormonal regulation of transcription of the gene for $P C K 1$ have been extensively studied previously, a better understanding of the regulation and function of bovine $P C K 1$ was sought by using specific primers to determine a detailed transcription profile in 9 different tissues. The potential splice variants and $P C K 1 \mathrm{mRNA}$ expression were investigated in various tissues of Chinese bulls and cows, to provide an explanation for the regulation of the tissue-dependent functions of the PCK1 gene.

\section{MATERIAL AND METHODS}

\section{Tissue collection and RNA isolation}

Tissue samples (heart, liver, spleen, lung, kidney, skeletal muscle, ovary, testis and sperm) were collected from 10 Chinese Holstein cattle (8 bulls and 2 cows) at the slaughter house at Jinan, Shandong Province, China. Tissues were immediately frozen in liquid nitrogen and kept at $-80^{\circ} \mathrm{C}$. Total RNA was isolated from tissues using an RNAsimple Total RNA kit (Tiangen), following the manufacturer instructions. RNA concentrations were measured with a Biophotometer (Eppendorf), and RNA quality was monitored by visualization of ethidium bromide-stained bands in $1 \%$ agarose gels after electrophoresis. Samples were stored at $-80^{\circ} \mathrm{C}$ before use.

\section{RT-PCR analysis}

First-strand cDNA was synthesized from 3-5 $\mu$ g total RNA using the RevertAid ${ }^{\mathrm{TM}}$ First Strand cDNA Synthesis kit (Fermentas) according to the manufacturer's instructions. A pair of specific primers (Table 1) were designed using PRIMER PREMIER 5.0 software and used to amplify the coding region spanning the 5'-untranslated and 3'-untranslated regions (UTR) of the bovine PCK1 gene (GenBank accession No. NM_174737). For subsequent PCR amplification, $2 \mu \mathrm{L}$ reverse-transcribed cDNA were combined with $10 \mu \mathrm{L}$ Hi-Fidelity Taq buffer, $0.1 \mu \mathrm{L}$ Hi-Fidelity Taq (Invitrogen, USA), $2.5 \mu \mathrm{M}$ oligonucleotide mixture, $0.5 \mu \mathrm{M}$ $\mathrm{MgSO}_{4}$ and $2.5 \mu \mathrm{M}$ dNTPs in a final volume of $25 \mu \mathrm{L}$. PCR was performed as follows: $94^{\circ} \mathrm{C}$ for $5 \mathrm{~min} ; 35$ cycles of $94^{\circ} \mathrm{C}$ for $30 \mathrm{~s}, 60^{\circ} \mathrm{C}$ for $1 \mathrm{~min}$ and $72^{\circ} \mathrm{C}$ for $1.5 \mathrm{~min}$; and $72^{\circ} \mathrm{C}$ for 10 min. DNA bands were separated by $1 \%$ agarose gel electrophoresis and eluted using the Gel Extraction kit (Biomiga, USA).

\begin{tabular}{llc} 
Table 1. Bovine $P C K 1$ and $\beta$-actin & gene primers. & Length (bp) \\
\hline Name & Sequence (5'-3') & 1980 \\
\hline$P C K 1$ cDNA & F: CTGACCTGATCGTCCAAAGAG & 173 \\
$\beta$-actin & R: CTGGTGCGTTGATGGATTG & \\
\hline
\end{tabular}




\section{Clone sequencing and AS identification}

The PCR products were subcloned into the pEASY-T3 cloning vector (TaKaRa, Dalian, China) immediately after purification, which was transformed into DH5 $\alpha$ competent cells. Subsequently, the cells were propagated in LB medium overnight at $37^{\circ} \mathrm{C}$. The plasmids were then purified using the Endo-free Plasmid Mini kit II (Omega, USA), and the cDNA inserts were sequenced with the ABI PRISMTM 3730 DNA Sequencer (Applied Biosystems) and Big Dye terminator v3.1 Sequencing kit (BGI, China). The splice variants were confirmed by the method described in a previous report (Huang et al., 2011).

Multiple sequence alignments were performed using DNAMAN v5.2.2 software (Lynon Biosoft) for the identification of possible splice variants. The protein secondary structure of identified splice variants was predicted using SWISS-MODEL (http://swissmodel.expasy.org/).

\section{RESULTS AND DISCUSSION}

\section{Expression of PCK1 mRNA in various tissues in cattle}

RT-PCR results showed that bovine $P C K 1$ mRNA expression was tissue-specific (Figure 1). The $P C K 1$ gene was highly expressed in liver, kidney, ovary and testis tissues, while expression abundance was low in the heart, spleen, sperm and lung tissues. No PCK1 mRNA was detected in skeletal muscle. The high expression of $P C K 1$ in liver and kidney is consistent with the fact that $P C K 1$ is involved in gluconeogenesis and glyceroneogensis in liver and the control of acid/base balance in kidney in many mammalian species. There is recent evidence that PCK1 is closely related to reproduction and female fertility in the mouse (Hanson and Hakimi, 2008). However, the role of PCK1 in the ovary and testis is unclear, but it most likely participates in either gluconeogenesis or glyceroneogenesis or reproduction and fertility in the cow.

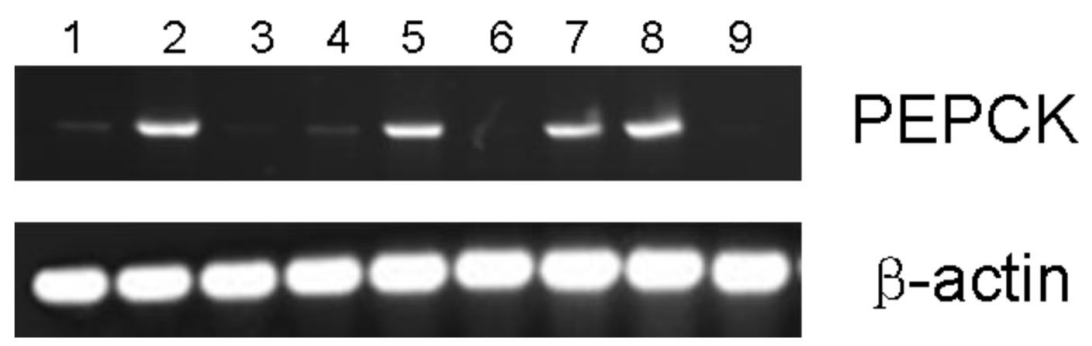

Figure 1. Expression pattern of PCK1 mRNA in various tissues in cattle. Lane 1 = heart; lane 2 = liver; lane $3=$ spleen; lane $4=$ lung; lane $5=$ kidney; lane $6=$ skeletal muscle; lane $7=$ ovary; lane $8=$ testis; lane $9=$ sperm.

\section{Identification of the bovine PEPCK transcript variants}

After the amplification of the full-length $P C K 1$ transcript with specific primers for $P C K 1$ mRNA reference sequence (Table 1) using cDNA from mammary tissues as templates, the PCR product of approximate $1.9 \mathrm{kbp}$ in size was found in 7 tissues. It was purified, subcloned and sequenced. Comparing the sequence obtained from each sample with the bovine 
PCK1 genomic reference sequence (GenBank accession No. AC_000170) and the PCK1 mRNA reference sequence (GenBank accession No. NM_174737.2), five novel splice variants of the bovine PCK1 gene, named PCK1-AS1-PCK1- $\bar{A} S 5$ (sequences submitted to NCBI, GenBank accessions: JQ733516-JQ733520), were detected (Figure 2). The result showed that the expression of PCK1 AS was tissue dependent (Table 2). The full-length transcript, designated as $P C K 1$-complete, was found in 6 tissues (Figure 1 and Table 2).

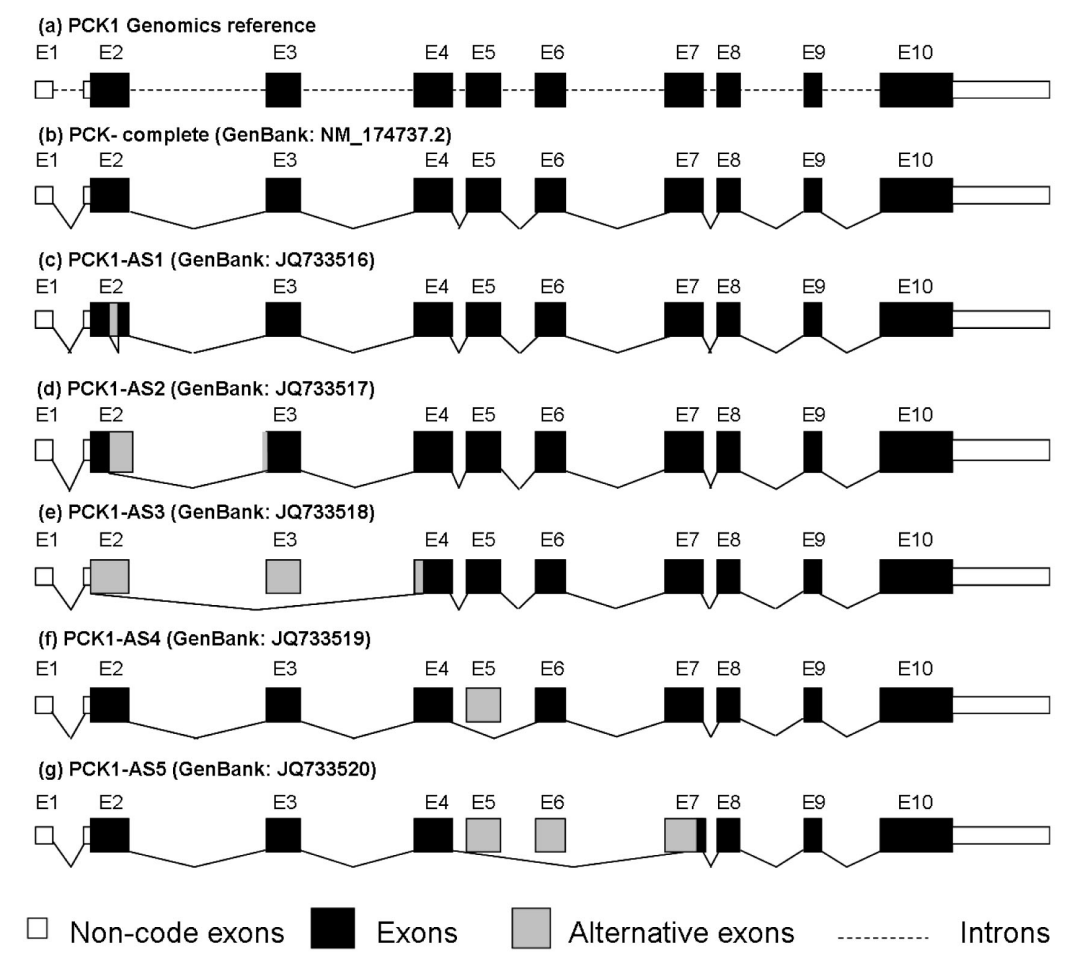

Figure 2. Genomic structure and alternative splicing patterns of the bovine PCK1 gene.

Table 2. Splice variants of the $P C K 1$ gene in the tissues of bull and cow.

\begin{tabular}{lccc}
\hline PCK1 splice variants & Length $(\mathrm{bp})$ & Tissue & Putative protein (aa) \\
\hline PCK1-complete & 1980 & Liver, kidney, testis, ovary, heart, lung & 622 \\
PCK1-AS1 & 1929 & Liver & 605 \\
PCK1-AS2 & 1891 & Liver & 546 \\
PCK1-AS3 & 1725 & Liver & 373 \\
PCK1-AS4 & 1792 & Testis & 246 \\
PCK1-AS5 & 1725 & Liver & 274 \\
\hline
\end{tabular}

In comparing with PCK1-complete, PCK1-AS1 had 51 bp deleted in exon 2 and encoded a putative protein with 605 amino acid (aa) residues. In PCK1-AS2, exon 2 and exon 3 of the PCK 1 gene were altered by the alternative 3 ' splice site and alternative 5 ' splice site, respectively. In the PCK1-AS2 transcript, a 90-bp sequence was spliced out, and a new initia- 
tor codon (ATG) was generated, which was predicted to encode a protein with 546 aa (Figure $3)$. The PCK1-AS3 was truncated from the $3^{\prime}$ end of exon 2 to the $5^{\prime}$ end of exon 4 of the PCK1-complete gene. The PCK1-AS2 had a total of $546 \mathrm{bp}$, where the normal initiator codon was spliced out. Therefore, this variant was predicted to encode a protein with 373 aa. Comparison of the predicted proteins encoded by the PCK1-AS3 and PCK1-complete splice variants revealed that the putative PCK1-AS3 isoform lost the domain of the PEP carboxykinase $\mathrm{N}$-terminal domain superfamily (Figures 4 and 5). The splicing pattern of PCK1-AS4 belongs to exon skipping. The PCK1-AS4 was spliced out in exon 5 of PCK1-complete, and the consequent amino acid sequence was changed with the early occurrence of the stop codon. The variant transcript was translated into a 246-aa protein which is apart of the PCK1-complete protein. The variant transcript of PCK1-AS5 was only translated into a 274-amino acid attributed to the splicing of exon 5, exon 6 and 5 ' end of exon 7.

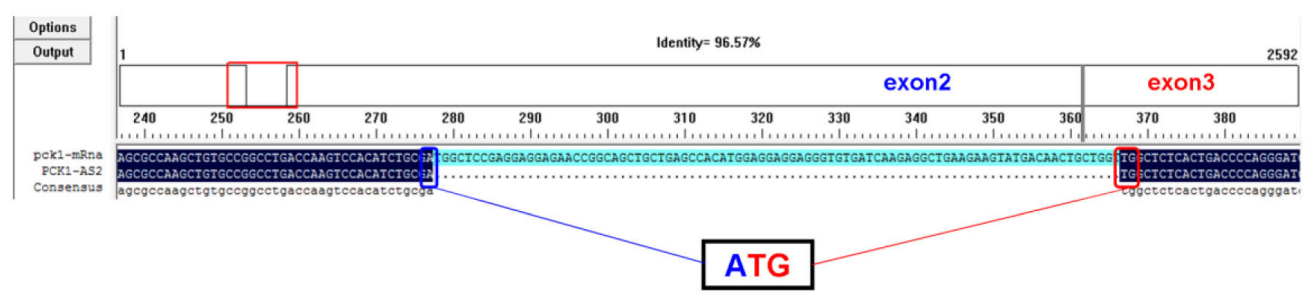

Figure 3. Alternative splicing pattern of the bovine $P C K 1$ - $A S 2$. In the $P C K 1-A S 2$, the exon 2 and exon 3 of the bovine $P C K 1-A S 2$ were altered by the alternative 3' splice site (A3SS) and alternative 5' splice site (A5SS), respectively. The last oligonucleotide of exon2 C-terminal was A and the N-terminal of exon3, just altered 5 oligonucleotides, starting with TG. Therefore, a new initiator codon (ATG) was generated.

Many genes encode a far greater number of proteins in all multicellular organisms by alternative splicing (Black, 2003). They can be expressed in a tissue-specific and temporally regulated manner under the control of pre-mRNA-binding proteins (Jelen et al., 2007). The mRNA and protein isoforms produced by alternative processing of primary RNA transcripts may differ in structure, function, localization or other properties (Matlin et al., 2005).

As shown in Figure 4, the protein three-dimensional structures and domains of identified splice variants were predicted using SWISS-MODEL (http://swissmodel.expasy.org/). Alternative splicing likely has a large impact on the function of the $P C K 1$ gene. The expression of PCK1-AS4 mRNA is testis-specific, and it encodes a 246-aa protein, which is different from the other isoform structures of the bovine $P C K 1$ gene through the alteration of the reading frame. Another four $P C K 1$ splice variants and $P C K$-complete were expressed in the liver. Alternative splicing is very frequent in the testis, which is the site of an extensive adult developmental program (Elliott and Grellscheid, 2006). Why is alternative splicing relatively abundant in the testis and liver? A relatively unique feature of the testis is that it is the site of an extensive adult developmental process. However, other factors may also be important in driving the extent of alternative splicing in the testis, since alternative splicing is also particularly high in the adult liver, which is terminally differentiated and not particularly complex in cell type content (Yeo et al., 2004). The present study demonstrates various bovine PCK1 splice variants existing in liver and testis tissues, which can explain in part the multiple functions of the $P C K 1$ gene. 


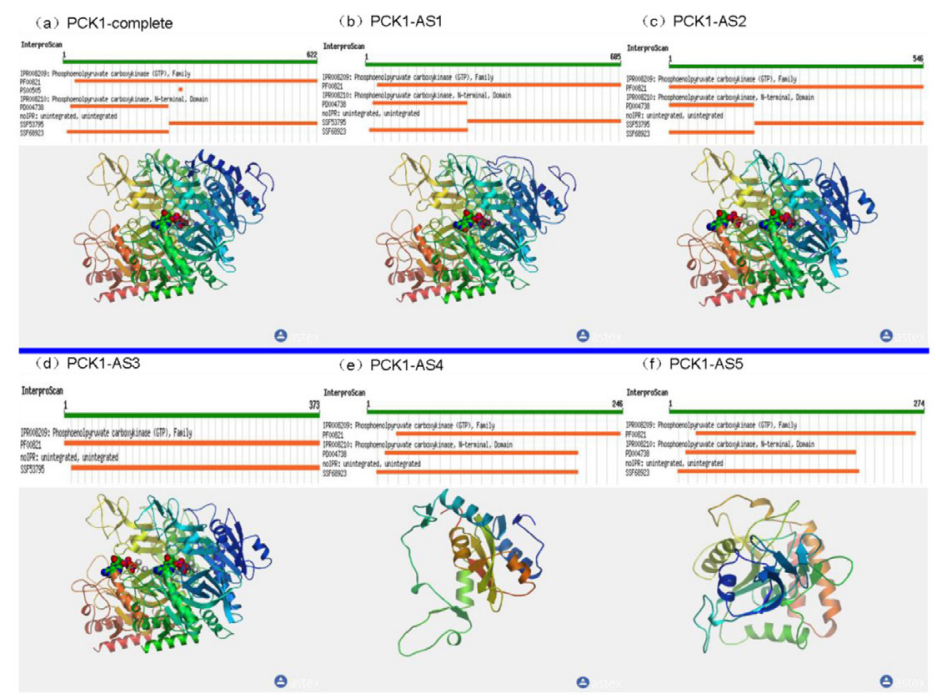

Figure 4. Putative protein structures and functional domains of the bovine $P C K 1$ splice variants identified. The transcript of PCK1-complete contains 5 domains: PR008209, phosphoenolpyruvate carboxykinase (GTP; PF00821: 29-622 PEPCK); IPR008209, phosphoenolpyruvate carboxykinase (GTP; PS00505: 284-292 PEPCK GTP); IPR008210, phosphoenolpyruvate carboxykinase, N-terminal (PD004738: 18-258 PEPCK_N); noIPR: unintegrated, unintegrated (SSF53795: 260-622 SSF53795); noIPR: unintegrated, unintegrated (SSF68923: 10259 SSF68923). The domain of PCPCK_N and SSF68923 in the PCK1-AS3 transcript was completely spliced out and the domain of PEPCK was partly spliced out. In PCK1-AS4 and PCK1-AS5, the domain of SSF53795 was completely lost and the domain of PEPCK was partly spliced out.

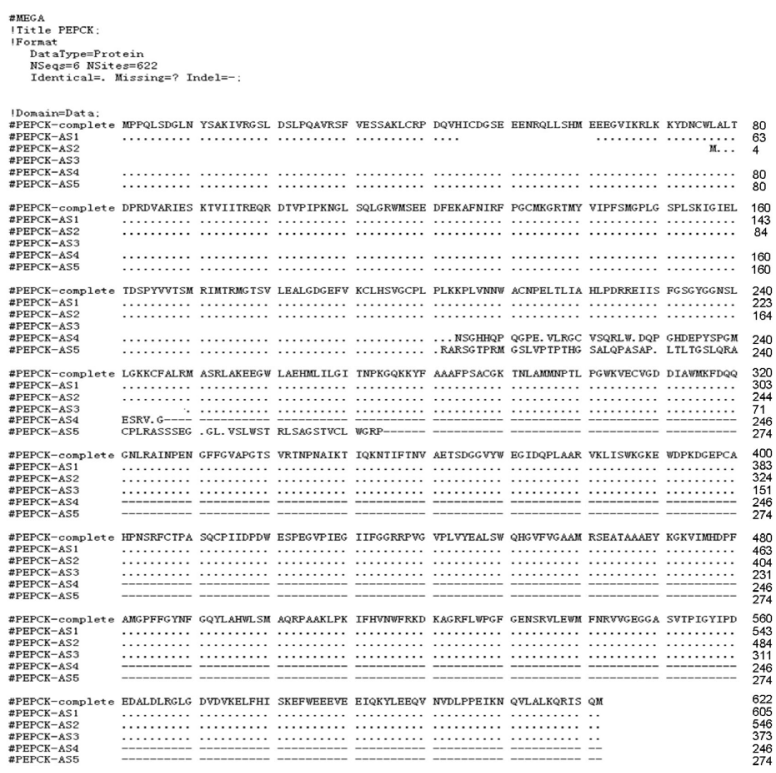

Figure 5. Multiple alignments of the predicted amino acid sequences of the bovine PCK1 transcript variants. $()=$. same amino acid when PCK1 transcript as compared to the PCK1-complete; $(-)=$ end of translation. 
In conclusion, our findings contribute to a better understanding of the complex bovine PCK1 gene. Considering the functional domains of the PCK1 protein, it is very likely that these splice variants affect the function of the protein considerably and that alternative splicing is one of the mechanisms by which the diverse functions of the bovine PCK1 gene are regulated.

\section{ACKNOWLEDGMENTS}

Research supported by the National Natural Science Foundation of China (\#31000543 and \#31271328), Support Program of the Ministry of Science and Technology, P.R. China (\#2011BAD19B02, \#2011BAD19B04 and \#2011BAD28B02), Major Project of National Transgene in China (\#2011ZX08007-001), Program of National Cow Industrial Technology System (CARS-37), and Project of Agricultural Fine Breed from the Department of Science and Technology of Shandong Province (\#2010LZ10-02).

\section{REFERENCES}

Agca C, Greenfield RB, Hartwell JR and Donkin SS (2002). Cloning and characterization of bovine cytosolic and mitochondrial PEPCK during transition to lactation. Physiol. Genomics 11: 53-63.

Black DL (2003). Mechanisms of alternative pre-messenger RNA splicing. Annu. Rev. Biochem. 72: 291-336.

Burgess SC, He T, Yan Z, Lindner J, et al. (2007). Cytosolic phosphoenolpyruvate carboxykinase does not solely control the rate of hepatic gluconeogenesis in the intact mouse liver. Cell Metab. 5: 313-320.

Chacko E and Ranganathan S (2009). Genome-wide analysis of alternative splicing in cow: implications in bovine as a model for human diseases. BMC Genomics 10 (Suppl 3): S11.

Chakravarty K and Hanson RW (2007). Insulin regulation of phosphoenolpyruvate carboxykinase-c gene transcription: the role of sterol regulatory element-binding protein 1c. Nutr. Rev. 65: S47-S56.

Chakravarty K, Cassuto H, Reshef L and Hanson RW (2005). Factors that control the tissue-specific transcription of the gene for phosphoenolpyruvate carboxykinase-C. Crit. Rev. Biochem. Mol. Biol. 40: 129-154.

Croniger CM, Olswang Y, Reshef L, Kalhan SC, et al. (2002). Phosphoenolpyruvate carboxykinase revisited: Insights into its metabolic role. Biochem. Mol. Biol. Educ. 30: 14-20.

Elliott DJ and Grellscheid SN (2006). Alternative RNA splicing regulation in the testis. Reproduction 132: 811-819.

Hakimi P, Yang J, Casadesus G, Massillon D, et al. (2007). Overexpression of the cytosolic form of phosphoenolpyruvate carboxykinase (GTP) in skeletal muscle repatterns energy metabolism in the mouse. J. Biol. Chem. 282: $32844-$ 32855.

Hanson RW and Patel YM (1994). P-Enolpyruvate Carboxykinase: The Gene and the Enzyme. John Wiley and Sons, New York, 203-281.

Hanson RW and Hakimi P (2008). Born to run; the story of the PEPCK-Cmus mouse. Biochimie 90: 838-842.

Huang JM, Wang ZY, Ju ZH, Wang CF, et al. (2011). Two splice variants of the bovine lactoferrin gene identified in Staphylococcus aureus isolated from mastitis in dairy cattle. Genet. Mol. Res. 10: 3199-3203.

Jelen N, Ule J, Zivin M and Darnell RB (2007). Evolution of Nova-dependent splicing regulation in the brain. PLoS Genet. 3: $1838-1847$.

Johnson JM, Castle J, Garrett-Engele P, Kan Z, et al. (2003). Genome-wide survey of human alternative pre-mRNA splicing with exon junction microarrays. Science 302: 2141-2144.

Liu K, Ba X, Yu J, Li J, et al. (2006). The phosphoenolpyruvate carboxykinase of Mycobacterium tuberculosis induces strong cell-mediated immune responses in mice. Mol. Cell Biochem. 288: 65-71.

Maniatis T and Tasic B (2002). Alternative pre-mRNA splicing and proteome expansion in metazoans. Nature 418: 236243.

Matlin AJ, Clark F and Smith CW (2005). Understanding alternative splicing: towards a cellular code. Nat. Rev. Mol. Cell Biol. 6: 386-398.

Yeo G, Holste D, Kreiman G and Burge CB (2004). Variation in alternative splicing across human tissues. Genome Biol. 5: R74. 